\title{
Carbeto de Silício como Material Base para Sensores MEMS de Uso Aeroespacial: Uma Visão Geral
}

\section{Silicon Carbide as Base Material for MEMS Sensors of Aerospace Use: An Overview}

Fraga, M.A. ${ }^{1}$, Pessoa, R. S. ${ }^{2}$, Massi, M. ${ }^{3}$, Maciel, H. S. ${ }^{2}$

\author{
${ }^{1}$ Departamento de Ensino Geral - FATEC-SP/ CEETEPS, São Paulo, SP \\ e-mail: fraga.mariana80@yahoo.com.br \\ ${ }^{2}$ Instituto de Pesquisa e Desenvolvimento, IP\&D/ UNIVAP, São José dos Campos, SP \\ e-mail: rodrigospessoa@gmail.com; homero@univap.br \\ ${ }^{3}$ Instituto de Ciência e Tecnologia, ICT/ UNIFESP, São José dos Campos, SP \\ e-mail: massi.marcos@gmail.com
}

\section{RESUMO}

Este artigo discute o emprego do carbeto de silício ( $\mathrm{SiC}$ ), na forma de substrato e filme fino, em sensores MEMS (MicroElectro-Mechanical Systems) para aplicações em ambientes sujeitos a condições extremas, especialmente no setor aeroespacial. As propriedades físicas e químicas do $\mathrm{SiC}$ que o tornam um material adequado para dispositivos eletrônicos e sensores são descritas. Os conceitos, evolução e aplicações da tecnologia MEMS são apresentados. Uma visão geral sobre o estágio atual de desenvolvimento de sensores MEMS baseados em SiC e uma análise das pesquisas realizadas nesta área no exterior e no Brasil, tanto nas universidades quanto nas indústrias, são também apresentad as. Os recentes avanços alcançados, as dificuldades encontradas e o impacto dessas pesquisas são discutidos, bem como as perspectivas para um futuro próximo.

Palavras-chave: carbeto de silício, materiais semicondutores, micro-sensores, aplicações aeroespaciais.

\section{ABSTRACT}

This paper discusses the use of silicon carbide ( $\mathrm{SiC}$ ), in bulk and thin-film form, in MEMS (Micro-Electro-Mechanical Systems) sensors for extreme environment applications, especially in aerospace. The physical and chemical properties of $\mathrm{SiC}$ that make it a suitable material for electronic devices and sensors are described. Concepts, developments and applications of MEMS technology are presented. An overview of the current stage of development of SiC-based MEMS sensors and an analysis of research conducted in this area in Brazil and abroad, both in universities and industries are also presented. The recent progress made, difficulties encountered and the impact of these investigations are discussed as well as the outlook for the near future.

Keywords: silicon carbide, semiconductor materials, microsensors, aerospace applications.

\section{INTRODUÇÃO}

Elevada variação de temperatura, radiação intensa e gases altamente corrosivos são condições prejudiciais para a aquis ição de dados na forma de sinais elétricos. No entanto, nas últimas décadas, o desejo de explorar o espaço tem impulsionado pesquisas sobre o desenvolvimento de sensores capazes de suportar ambientes com faixa de temperatura entre $-215^{\circ} \mathrm{C}$ a $450^{\circ} \mathrm{C}$ e de pressão da ordem de alto vácuo até 1000 bar $[\underline{1}, \underline{2}]$. Nas primeiras missões espaciais, dispositivos de medições indiretas ou aparelhos volumosos eram empregados. Os custos elevados, a área ocupada e o peso desses equipamentos tornaram-se uma problemática. Até que nos anos de 1980, surgiu a tecnologia de sistemas micro-eletromecânicos conhecida como MEMS (Micro-Electrical-Mechanical Systems) ou MST (Microsystem Technologies). Esta tecnologia possibilitou a produção de dispositivos com componentes, cujas dimensões variam de um micrometro até um milímetro, construídos a partir de diversas técnicas de microfabricação que foram desenvolvidas para circuitos integrados.

Os fatores que impulsionaram a tecnologia MEMS foram os mesmos da microeletrônica: miniaturização, confiab i- 
lidade, produção em massa e redução de custos. A integração de componentes eletrônicos e mecânicos num único "chip" permitiu a implementação de dispositivos, cada vez menores, mais leves, mais baratos e com maior funcionalidade. A combinação desses fatores gerou impactos significativos em diferentes setores industriais, tais como: automotivo, aeroespacial, automação industrial e biomédico.

Dispositivos MEMS podem ser fabricados a partir de uma variedade de materiais (semicondutores, polímeros, metais e cerâmicos), que empregam diferentes princípios físicos (piezoelétrico, piezoresistivo, eletrostático, capacitivo, térmico e eletromagnético), para a detecção e atuação, num vasto campo de aplicações como, por exemplo, microfluídica, navegação inercial, radiofrequência (RF) e ótica. Para aplicações em sensoriamento, o mecanismo de transdução mais usado é o mecânico-elétrico dos seguintes tipos: piezoelétrico, piezoresistivo e capacitivo []] . A Figura 1 mostra alguns tipos de sensores e atuadores MEMS disponíveis comercialmente, além da emergente aplicação na área de micro-displays [4].

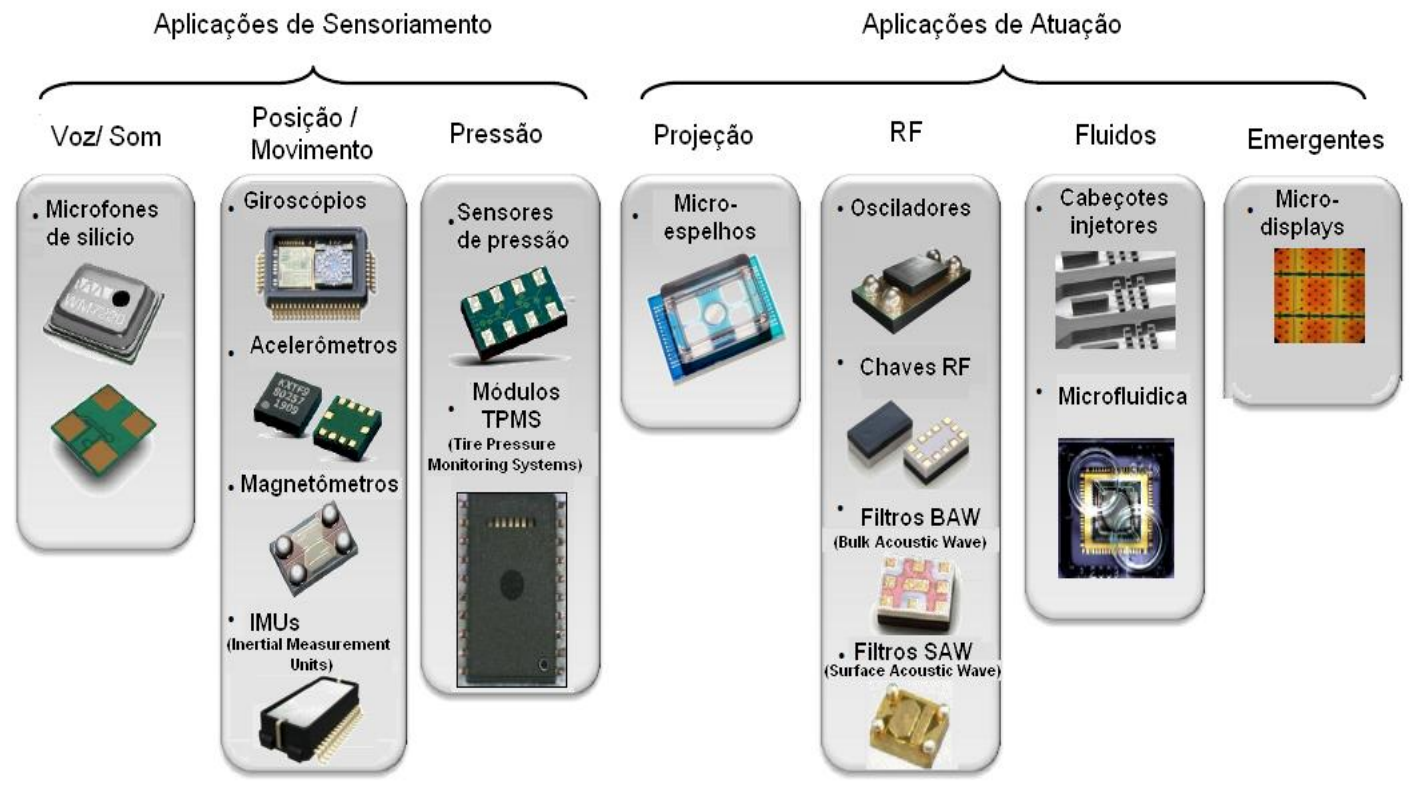

Figura 1: Alguns sensores e atuadores MEMS (adaptado de [ㄴ]).

Assim como a tecnologia de circuitos integrados, a tecnologia de fabricação de sensores MEMS está baseada no silício que é o material semicondutor mais estudado e utilizado na indústria . Sabe-se que o silício é sensível a ambientes extremos como, por exemplo, radiação intensa, atmosferas corrosivas e temperaturas elevadas (maiores que $175^{\circ} \mathrm{C}$ ) [5]. Entretanto, diversas aplicações de diferentes setores, como os ilustrados na Figura 2, necessitam de sensoriamento em temperaturas elevadas, onde seria inviável ou ineficiente o uso de dispositivos MEMS baseados neste material. Observase que tais aplicações demandam alguns tipos de sensores, principalmente de pressão.

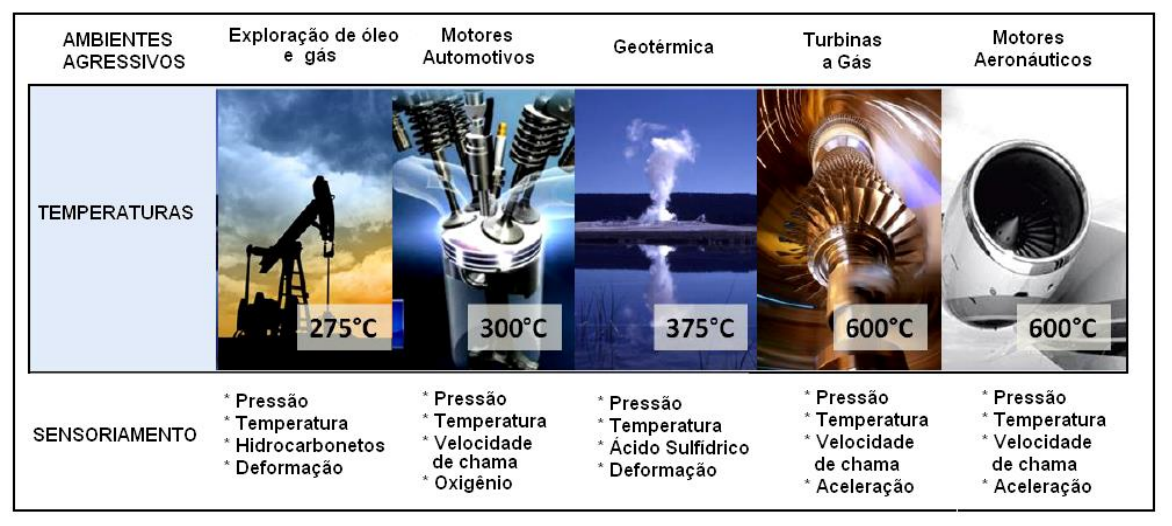

Figura 2: Aplicações em temperaturas elevadas que requerem diferentes tipos de sensores [్]. 
Diante disso, a academia e a indústria se depararam com as seguintes questões: Seria possível viabilizar o uso de sensores MEMS baseados em silício, em ambientes extremos utilizando encapsulamentos que os isolasse do meio? Ou o melhor seria substituir o silício como elemento de transdução? Quais materiais poderiam substituí-lo? Como adaptar os processos de microfabricação estabelecidos para o silício para esses materiais?

Esses questionamentos motivaram e ainda motivam diversas pesquisas tanto sobre encapsulamento quanto sobre os materiais que constituem os elementos de transdução. O setor aeroespacial tem sido um dos que impulsiona essas pesquisas dado que, como mencionado anteriormente, as aplicações espaciais típicas expõem os sensores a condições ambientais adversas como alta radiação, temperaturas extremas, pressões baixas ou elevadas, choques, vibrações e atmosferas corrosivas.

(a)

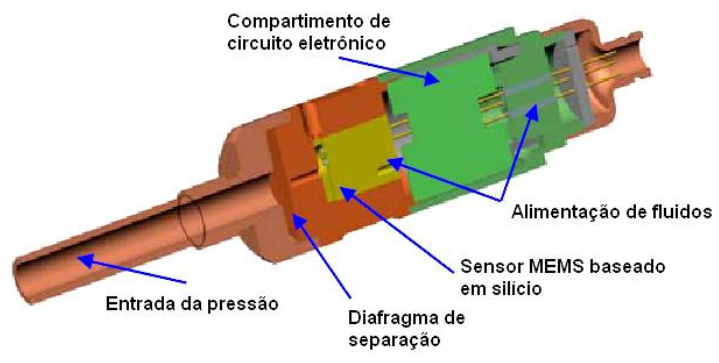

(c)

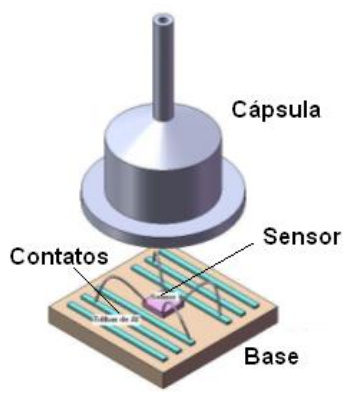

(b)

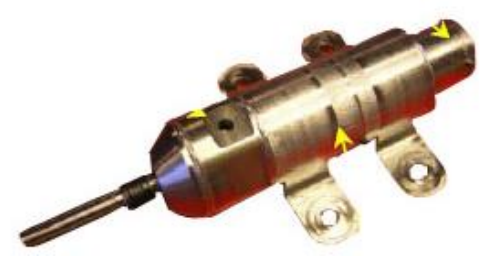

(d)

Figura 3: Ilustrações: (a) e (b) Sensor de pressão de silício encapsulado para aplicações em ambientes extremos (adaptado de [8]), (c) e (d) sensor encapsulado para aplicações em ambientes comuns.

A literatura recente tem mostrado que o encapsulamento de sensores MEMS baseados em silício, para aplicações em temperaturas elevadas, é realizado de forma que o fluido transmita a pressão aos elementos sensores que estão afastados do ponto de medição. Alguns sensores MEMS encapsulados por esse método estão sendo aplicados no sensoriamento de pressão em motores de combustão [7]. Contudo, essa tecnologia, aumenta significativamente o tamanho, o peso e os custos dos dispositivos. Na Figura 3 são mostradas ilustrações de encapsulamentos de sensores MEMS de silício para ambientes extremos e para ambientes comuns []].

No desenvolvimento de sensores MEMS para suportar ambientes extremos, a opção de substituir o silício por outros materiais semicondutores de elevada largura de banda proibida ("band-gap") tem se mostrado viável, pois apresenta menor custo por não requerer um processo de encapsulamento robusto [9].

Nos últimos anos, a agência espacial americana (NASA) tem divulgado estudos sobre materiais alternativos ao silício, que podem ser usados em micro-dispositivos eletrônicos e sensores MEMS para aplicações aeroespaciais [5] . O carbeto de silício $(\mathrm{SiC})$ tem sido posicionado como um dos materiais mais promissores devido à sua estabilidade química e térmica, boas propriedades mecânicas (elevada rigidez e dureza), resistência a radiação, além de ser compatível com a maioria dos processos de microfabricação que foram estabelecidos para o silício. Sensores de pressão e acelerômetros MEMS baseados em SiC (na forma de substrato e de filme fino) têm sido testados com sucesso em temperaturas de até $600^{\circ} \mathrm{C}[10]$ 
Outro aspecto a ser considerado é que a aplicação aeroespacial de dispositivos microfabricados é diferente em comparação com os de aplicação terrestre, pois a produção em larga escala não é um requisito principal. Muitos sistemas aeroespaciais são únicos ou produzidos em pequeno número. Geralmente, cada sensor é desenvolvido especificamente para se integrar a um dado veículo espacial. Neste caso, a confiabilidade é a questão principal. O sensor, além de executar suas funções no sistema, deve funcionar por tempo prolongado e sem falhas.

Embora os sensores MEMS baseados em SiC apresentem desempenho satisfatório quando submetidos a temperaturas elevadas, altas pressões e ambientes corrosivos, a tecnologia de fabricação deles ainda não está amadurecida. Existem alguns desafios tecnológicos a serem superados, especialmente relacionados à síntese do $\mathrm{SiC}$ e à reprodutibilidade dos sensores.

Este artigo apresenta uma visão geral sobre a evolução e o estágio atual da tecnologia de sensores MEMS baseados em SiC, ao mesmo tempo que discute os principais desafios para usá-los em aplicações aeroespaciais. Para isso é importante apresentar em detalhes das características físicas e químicas do $\mathrm{SiC}$, destacando suas vantagens com relação ao silício. Também são apresentadas algumas pesquisas realizadas no Brasil e no exterior sobre o desenvolvimento de protótipos de sensores MEMS baseados em SiC, bem como as perspectivas para viabilizar sua comercialização.

\section{O POTENCIAL DO SIC PARA SENSORES MEMS DE USO AEROESPACIAL}

A seleção de materiais adequados pode ser um fator determinante para estimar se um dispositivo MEMS funcionará como projetado em ambiente espacial. Os materiais devem ser capazes de suportar temperaturas elevadas e radiação intensa sem sofrer degradação [11].

Como já mencionado, a tecnologia MEMS está baseada no silício que é um material de estreita banda de energia proibida, da ordem de $1,1 \mathrm{eV}$, o que limita sua aplicação em ambientes extremos. Na literatura, é bem documentado como a temperatura afeta os mecanismos de condução no silício limitando o seu uso em dispositivos eletrônicos e MEMS quando operados em temperaturas acima de $175^{\circ} \mathrm{C}$ [12] .

O efeito da radiação sobre o silício é também bem conhecido, principalmente devido aos testes realizados com circuitos eletrônicos integrados. A Figura 4 mostra uma visão geral do efeito da radiação sobre materiais e dispositivos [13]. A interação entre a radiação e o silício resulta em ionização e danos de deslocamento atômico. Estudos têm demonstrado como essas alterações na estrutura do silício afetam as características elétricas desses circuitos [14]. Entretanto, para avaliar a influência da radiação sobre o desempenho de sensores MEMS é necessário conhecer como as propriedades mecânicas do silício são afetadas.

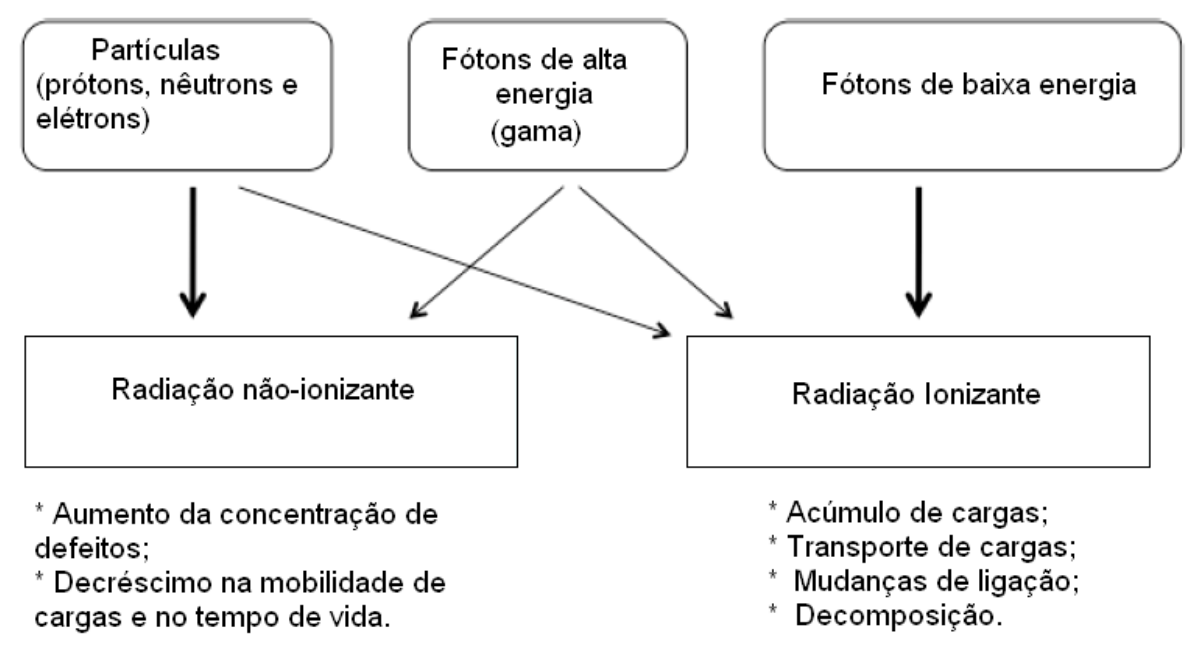

Figura 4: Resumo dos efeitos de degradação induzidos por radiação, ignorando efeitos trans itórios [13].

Em 2011, H. R. Shea publicou um artigo sobre o efeito da radiação em dispositivos MEMS baseados em silício [13]. De acordo com os resultados obtidos, ele concluiu que a degradação dos dispositivos devido à radiação é uma complexa interação de alterações nos materiais e no princípio físico de atuação (piezo, capacitivo, eletromagnético e eletrotérmico e ótico). Por exemplo, não existe quase nenhuma mudança no módulo de elasticidade do silício quando submetido à radiação gama. Portanto, não se espera que a frequência de ressonância de um "cantilever" ou da massa inercial de silício de um acelerômetro MEMS possa se alterar permanentemente após essa irradiação. No entanto, a radiação gama pode levar ao armazenamento de cargas na camada do dielétrico do dispositivo. Logo, se o efeito capacitivo é utilizado 
para detectar a frequência de ressonância, uma grande alteração dos parâmetros do dispositivo pode ser observada. Se um princípio ótico (como no vibrômetro laser Doppler) for usado, nenhuma mudança será observada [13].

Os mecanismos do acúmulo de cargas nas camadas de materiais dielétricos de dispositivos MEMS devido à radiação têm sido o foco de várias pesquisas $[15, \underline{16}$. Isto porque, assim como nos circuitos integrados, camadas de óxidos ou nitretos (geralmente $\mathrm{SiO}_{2}$ ou $\mathrm{Si}_{3} \mathrm{~N}_{4}$ ) são usadas na microfabricação dos dispositivos e, após os processos, não são totalmente removidos para que atuem como isolante elétrico. A Tabela 1 resume o efeito do acúmulo de cargas no dielétrico em função do princípio de atuação (ou transdução) [16].

Tabela 1: Principais modos de falha devido ao acúmulo de cargas no dielétrico em função do princípio de atuação MEMS [16].

\begin{tabular}{c|c|c}
\hline $\begin{array}{c}\text { PRINCÍPIO FÍSICO DE } \\
\text { ATUAÇÃo }\end{array}$ & $\begin{array}{c}\text { SENSIBILIDADE AO } \\
\text { ACÚMULO DE CARGAS } \\
\text { NO DIELÉTRICO }\end{array}$ & $\begin{array}{c}\text { FALHAS DEVIDO AO ACÚMULO } \\
\text { DE CARGAS }\end{array}$ \\
\hline Eletrostático/ capacitivo & Alta & Variação da capacitância \\
\hline Magnético & Muito fraca & Variação da tensão de ruptura \\
\hline Piezo & Fraca a média & $\begin{array}{c}\text { Mudança na calibração (falhas são } \\
\text { devido à radiação não ionizante) }\end{array}$ \\
\hline Eletrotérmico & Muito fraca & Variação da tensão de ruptura \\
\hline
\end{tabular}

Nota-se que embora a radiação modifique algumas propriedades do silício grande parte do efeito da radiação sobre os dispositivos MEMS está associada à influência do acúmulo de cargas no dielétrico sobre o mec anismo de atuação. Os mecanismos piezo (piezoelétrico e piezoresistivo) são os princípios mais empregados no desenvolvimento de sensores MEMS para ambientes sujeitos a radiação porque são menos sensíveis que o capacitivo [15].

Diante da discussão sobre o uso de silício em dispositivos MEMS para ambientes extremos, as temperaturas elevadas e atmosferas corrosivas têm sido mostradas como fatores mais limitantes que a radiação. Tanto as pesquisas relacionadas ao estudo dos efeitos da temperatura quanto às relacionadas a ambientes corrosivos apontam para uma mesma direção: o uso de materiais semicondutores de elevada largura de banda proibida ("band gap") como o carbeto de silício $(\mathrm{SiC})$ com $\mathrm{E}_{\mathrm{g}}$ entre 2,3 e 3, $3 \mathrm{eV}$, nitreto de alumínio ( $\mathrm{AlN}$ ) com $\mathrm{E}_{\mathrm{g}}=6,2 \mathrm{eV}$, diamante com $\mathrm{E}_{\mathrm{g}}=5,45 \mathrm{eV}$ e óxido de zinco $(\mathrm{ZnO})$ com $\mathrm{E}_{\mathrm{g}}=3,37 \mathrm{eV}$, entre outros. A Tabela 2 resume algumas aplicações específicas em alta temperatura nas quais materiais semicondutores estão sendo testados como material base para sensores MEMS [17]. Entre os materiais de elevado "band gap", o SiC e o diamante são os mais estudados devido as suas excelentes propriedades físicas [17- $\underline{19}]$.

Tabela 2: Aplicações em temperaturas elevadas que requerem tecnologia de semicondutores (adaptado de [17]).

\begin{tabular}{c|c|c|c|c}
\hline SETOR & $\begin{array}{c}\text { APLICAÇÕES EM TEMPE- } \\
\text { RATURAS ELEV ADAS }\end{array}$ & $\begin{array}{c}\text { TEMPERATURA } \\
\text { MÁXIMA DE USO (ํ) })\end{array}$ & $\begin{array}{c}\text { TECNOLOGIA DE } \\
\text { SEMICONDUTORES } \\
\text { USADA }\end{array}$ & PERSPECTIVAS \\
\hline \multirow{2}{*}{ Automotivo } & $\begin{array}{c}\text { Suspensão e freio } \\
\text { Cilindros e escape }\end{array}$ & 250 & $\begin{array}{c}\text { Silício } \\
\text { Não há }\end{array}$ & $\begin{array}{c}\text { Materiais de elevado } \\
\text { "band-gap" }\end{array}$ \\
\hline Aeronáutico & Motor de turbina & 600 & Não há & $\begin{array}{c}\text { Materiais de elevado } \\
\text { "band-gap" }\end{array}$ \\
\hline $\begin{array}{c}\text { Exploração de } \\
\text { petróleo e gás }\end{array}$ & Perfuração de poços & 300 & $\begin{array}{c}\text { SOI (Silicon-On- } \\
\text { Isulator) }\end{array}$ & $\begin{array}{c}\text { Materiais de elevado } \\
\text { "band-gap" }\end{array}$ \\
\hline Espacial & Veículos espaciais & 550 & Não há & $\begin{array}{c}\text { Materiais de elevado } \\
\text { "band-gap" }\end{array}$ \\
\hline
\end{tabular}

Na Tabela 3 são comparadas algumas propriedades do silício com as do $\mathrm{SiC}$ (polítipos 3C-, 4H- e 6H-) e do diamante. 
Tabela 3: Comparação entre as propriedades dos polítipos do $\mathrm{SiC}(3 \mathrm{C}-$-, 4H- e 6H-) com as do silício e diamante [19].

\begin{tabular}{c|c|c|c|c|c|c}
\hline PROPRIED ADE & 3C-SiC & 4H-SiC & $\mathbf{6 H - S i C}$ & $\mathbf{S i}$ & GaAs & DIAMANTE \\
\hline Band gap (eV) & 2,36 & 3,23 & 3,00 & 1,11 & 1,42 & 5,50 \\
\hline $\begin{array}{c}\text { Tensão de ruptura } \\
\text { (MV/cm) }\end{array}$ & 1,0 & 3,0 a 5,0 & 3,0 a 5,0 & 0,3 & 0,4 & 10,0 \\
\hline $\begin{array}{c}\text { Condutividade térmica } \\
(\text { W/cm-K) }\end{array}$ & 3,6 & 3,7 & 4,9 & 1,3 & 0,46 & 20,0 \\
\hline $\begin{array}{c}\text { Módulo de elasticidade } \\
(\mathrm{GPa})\end{array}$ & 448 & 448 & 448 & 190 & 85,5 & 1035 \\
\hline Constante dielétrica & 9,7 & 9,7 & 9,7 & 11,7 & 13,1 & 5,0 \\
\hline
\end{tabular}

O SiC é o único composto binário de silício ( $\mathrm{Si}$ ) e carbono (C) conhecido. Ambos $\mathrm{Si}$ e $\mathrm{C}$ pertencem a elementos do grupo IV. Eles são delimitados por hibridizações do tipo $\mathrm{sp}^{3}$. Na forma de filme fino, o SiC pode ser encontrado na estrutura amorfa ou cristalina [18]. O carbeto de silício amorfo (a-SiC) favorece a formação de uma rede de tetraedros onde a fração C/Si tem uma forte influência sobre a rede.

A característica mais notável da estrutura do cristal de SiC é o seu politipismo, onde a estrutura cristalina apresenta um número de diferentes sequências de ordenação unidimensionais sem qualquer variação de estequiometria. Registros apontam que existem mais de 200 politipos do $\mathrm{SiC}$, sendo que os polítipos 3C-SiC, 4H-SiC e $6 \mathrm{H}-\mathrm{SiC}$ são os que apresentam melhores propriedades semicondutoras $[\underline{18}, \underline{19}]$. A unidade atômica e as estruturas do cristal de SiC são apresentados na Figura 5.

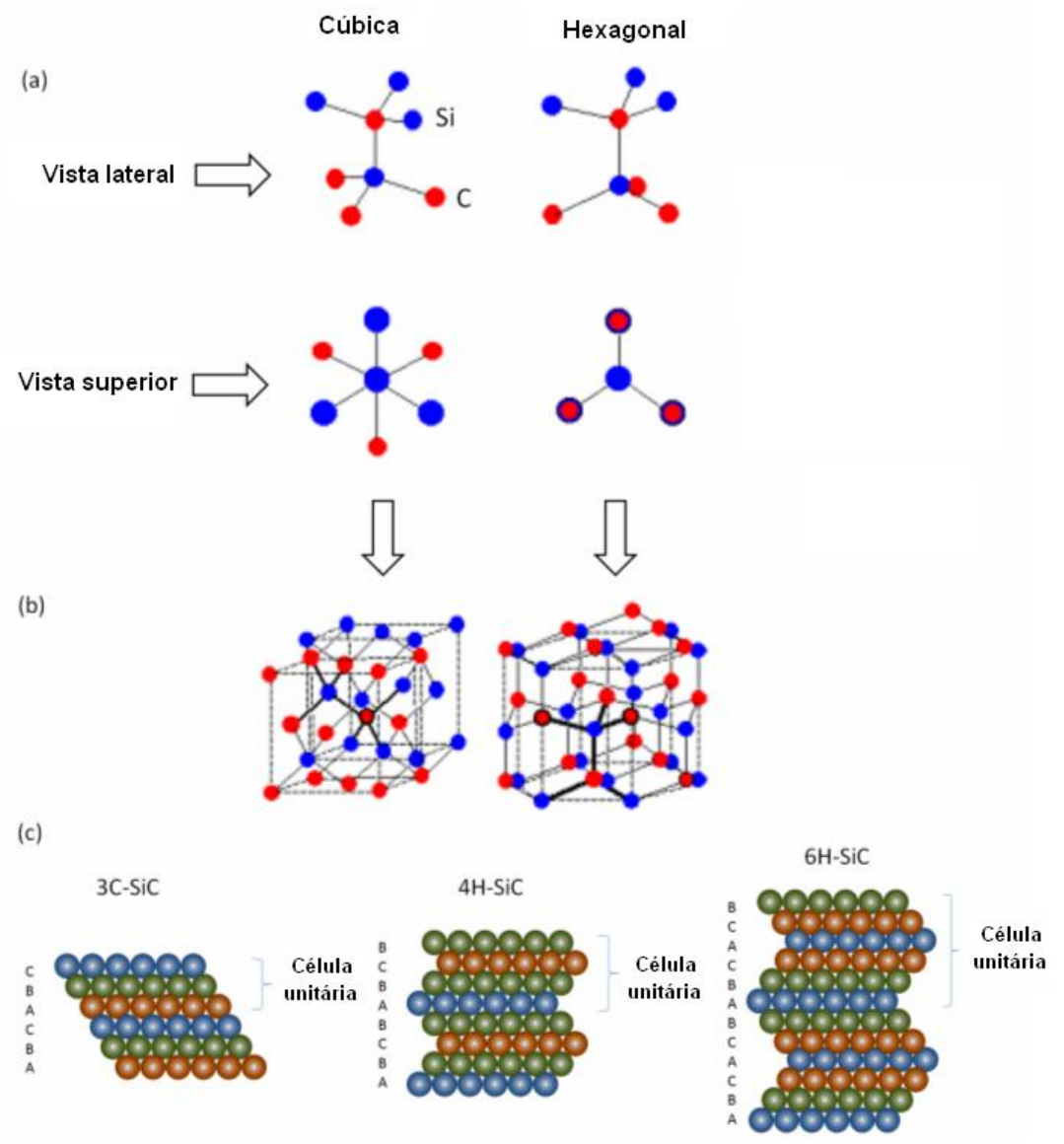

Figura 5: (a) Estrutura atômica do SiC. (b) Estrutura cristalina dos diferentes politipos do SiC. (c) Diferentes empilhamentos do $\mathrm{SiC}$ formando os mais comuns polítipos do $\mathrm{SiC}$ (adaptado de [19, 20]).

No entanto, tem sido demonstrado que o polítipo 3C-SiC tem melhores propriedades do que os $4 \mathrm{H}-\mathrm{SiC}$ e $6 \mathrm{H}-\mathrm{SiC}$. 
Por exemplo, 3C-SiC é o único polítipo do $\mathrm{SiC}$ com propriedades isotrópicas devido à simetria de cristal cúbico. Além disso, tem a mais alta saturação da velocidade de deriva e a maior mobilidade dos elétrons. No entanto, o crescimento do polítipo 3C-SiC de boa qualidade sobre substratos de Si é problemático devido à grande incompatibilidade entre os parâmetros das redes cristalinas $(\sim 20 \%)$ e a diferença entre os seus coeficientes de expansão térmica $(\sim 8 \%)$ a temperatura ambiente [19]. Assim como o diamante, o SiC é um semicondutor de elevado "band-gap" com propriedades físicas e elétricas superiores para substituir materiais semicondutores convencionais como o Si e o arseneto de gálio (GaAs) em aplicações MEMS (Tabela 3). O diamante é considerado o mais ideal dos semicondutores de elevado "band-gap" devido às suas excelentes propriedades eletrônicas e físicas. No entanto, tem uma estrutura atômica extremamente organizada, fato que dificulta sua dopagem. Também, embora as propriedades elétricas e mecânicas do SiC sejam inferiores às do diamante, em altas temperaturas (superiores a $300^{\circ} \mathrm{C}$ ) o $\mathrm{SiC}$ permanece estável enquanto o diamante tende a se tornar grafite [18]. Por fim, as tecnologias de processamento do $\mathrm{SiC}$ são similares as do silício o que o torna mais atrativo para uso em dispositivos eletrônicos e MEMS comerciais.

As características fundamentais do $\mathrm{SiC}$ que demonstram seu potencial para aplicações em dispositivos eletrônicos de alta potência e para temperaturas elevadas são:

- Tensão de ruptura elevada (dez vezes maior que a do silício): permite a fabricação de dispositivos que suportam grandes diferenças de potenciais;

- Alta saturação da velocidade de deriva dos elétrons: faz com que seja favorável para aplicações em dispositivos que operam em alta frequência;

- Elevado "band-gap": resulta em uma baixa concentração de portadores de cargas intrínsecos, o que permite alta temperatura de operação, sem excessiva corrente de fuga;

- Estabilidade térmica e química: é fundamental para a operação contínua em temperat uras elevadas ou ambientes quimicamente agressivos. Essa estabilidade pode ser exemplificada pela alta temperatura de sublimação do SiC na ordem de $2830^{\circ} \mathrm{C}[\underline{10}]$.

\section{TECNOLOGIA MEMS}

Nos últimos anos, o $\mathrm{SiC}$ tem se destacado como um material para dispositivos eletrônicos de alta potência e alta temperatura, tais como: termistores (até $1050^{\circ} \mathrm{C}$ ), tiristores (até $500^{\circ} \mathrm{C}$ ), diodos de potência (até $700^{\circ} \mathrm{C}$ ) e amplificadores operac ionais MOSFET (Metal-Oxide Semiconductor Field Effect Transistor) (até $600^{\circ} \mathrm{C}$ ) [1]].

Em termos de dispositivos MEMS, aplicações de grande impacto são os sensores de pressão piezoresistivos usados em motores de combustão e os sensores de gás desenvolvidos para monitoramento de emissões de gases poluentes e detecção de vazamento de hidrocarbonetos. Não obstante a outras aplicações, os sensores piezoresistivos baseados em $\mathrm{SiC}$ são os de maior potencial comercial dado que os sensores piezoresistivos, de pressão e aceleração, correspondem a 30\% do mercado mundial de sensores integrados [21]. A consolidação da tecnologia de sensores MEMS baseados em SiC possibilitará a ampliação do mercado por viabilizar o uso dos sensores em ambientes extremos.

De acordo com o NIST (National Institute of Standards and Technology), órgão do governo norte-americano que estabelece padrões de qualidade para produtos industriais, um sensor inteligente é definido como um único "chip", sem componentes externos, que inclui detecção, interfaceamento e processamento de sinais. Sensores baseados nesse conceito exploram a tecnologia MEMS em conjunto com eletrônica avançada e dispositivos sem fio que utilizam tec nologias de comunicação por RF [22]. Devido à sua dimensão reduzida e estrutura integrada, sensores MEMS podem ser facilmente incorporados em estruturas de materiais compósitos ou imprensados entre componentes metálicos para controle remoto sem fio e monitoramento via internet [23].

A natureza multidisciplinar da tecnologia MEMS está ilustrada na Figura 6. Nota-se que o projeto, fabricação e testes de dispositivos MEMS requerem a aplicação de princípios de física e química bem como de diferentes áreas da engenharia [24].

Embora o primeiro sensor de pressão de silício microusinado tenha sido fabricado em 1969, apenas no início dos anos de 1980 o potencial da tecnologia MEMS foi de fato reconhecido, com a fabricação e teste dos primeiros sensores integrados pela empresa Honeywell [25]. As indústrias nas áreas de microeletrônica e biomédica foram as primeiras a investir na concepção e implementação de diferentes tipos de sensores MEMS. Em 1992, a Universidade de Cornell estabeleceu o processo SCREAM (Single Crystal Reactive Etch and Metallization) que foi o primeiro processo desenvolvido especificamente para fabricação de dispositivos MEMS [26].

Os primeiros sensores MEMS piezoresistivos baseados em SiC foram fabricados no final dos anos de 1990. Desde então, diferentes sensores foram desenvolvidos baseados no substrato ou no filme fino deste material, o que resulta em duas configurações: piezoresistores de $\mathrm{SiC}$ sobre diafragma de $\mathrm{SiC}$ ou piezoresistores de $\mathrm{SiC}$ sobre diafragma de silício. Cada configuração apresenta vantagens e desafios pertinentes tanto a síntese do $\mathrm{SiC}$ bem como ao seu processamento 
para construção do sensor. Essas configurações serão discutidas mais adiante na próxima seção.

Nos últimos 20 anos, a tecnologia MEMS, saiu do campo da experimentação e com os avanços dos processos de microfabricação, consolidou-se como uma tecnologia chave para o desenvolvimento de sensores para diferentes aplic ações, especialmente automotivas. O mercado de dispositivos MEMS e os principais setores de aplic ação desses dispositivos são mostrados na Figura 7.

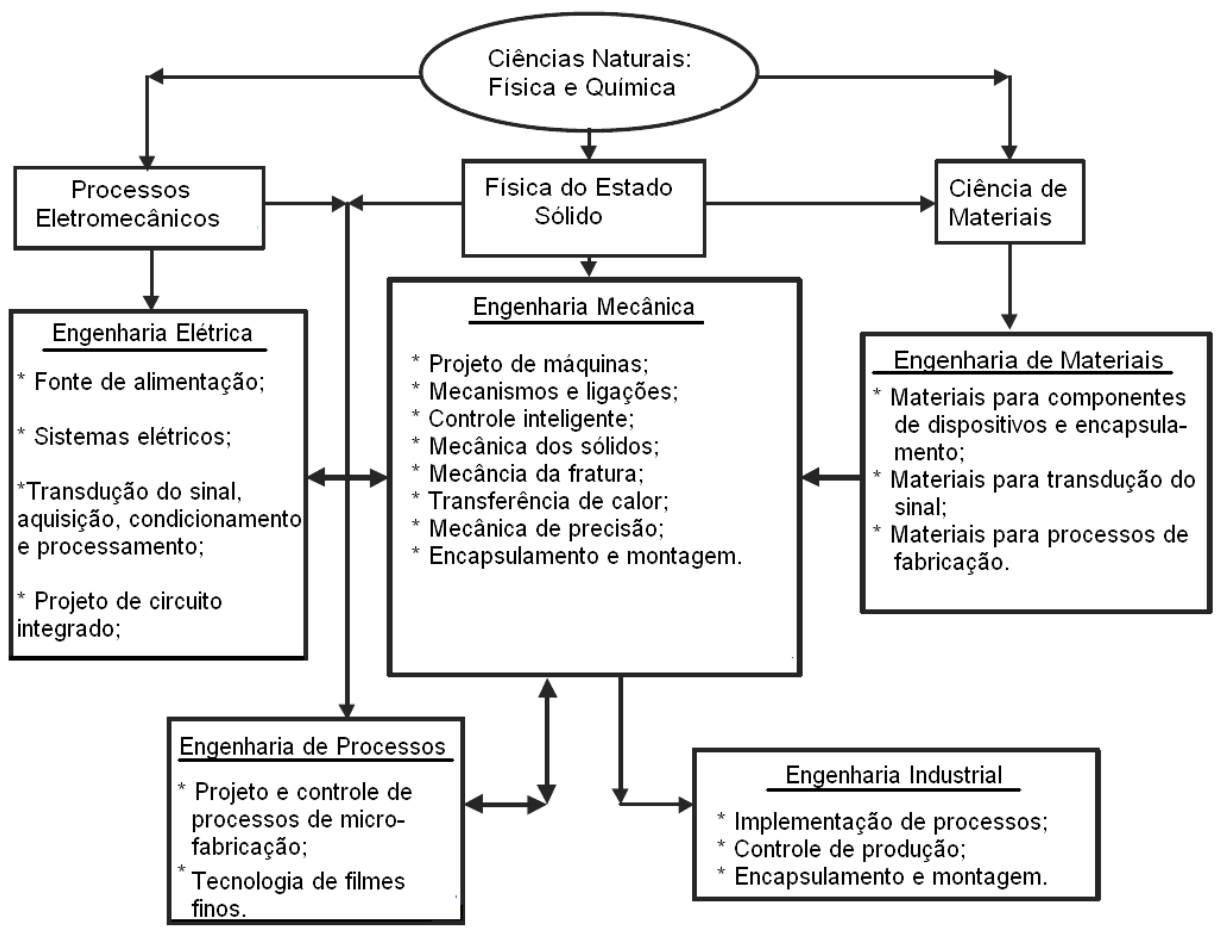

Figura 6: A multid isciplinaridade da tecnologia MEMS (adaptado de [24]).

\section{Mercado de MEMS por Aplicaçã̃o (em milhões de dólares americanos)}

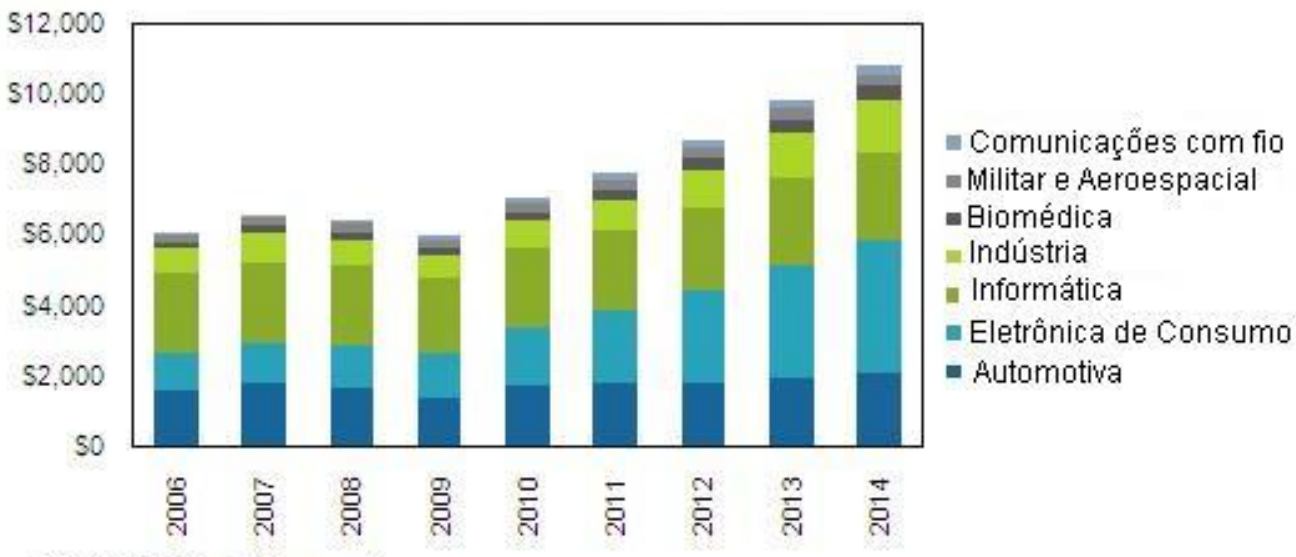

Fonte: /HS iSuppil Research

Figura 7: Mercado de Sensores MEMS por aplicação para o período de 2006-2014 [27].

Os avanços em encapsulamento, interconexões e integração e o surgimento de novas aplicações que demandam por sensores MEMS estão ilustrados na Figura 8. Hoje os sensores MEMS são uma realidade em vários tipos de aparelhos eletrônicos e já são associados ao conceito de poeira inteligente ( "smart dust") que são dispositivos minúsculos dotados de comunicação sem fio, capazes de medir diferentes grandezas físicas (como por exemplo, temperatura), para aplicações 
em monitoramento ambiental e de segurança [22]. Também surgiram os primeiros dispositivos NEMS (Nanoelectromechanical Systems) fabricados a partir da adaptação dos processos da tecnologia MEMS para escala nanométrica.

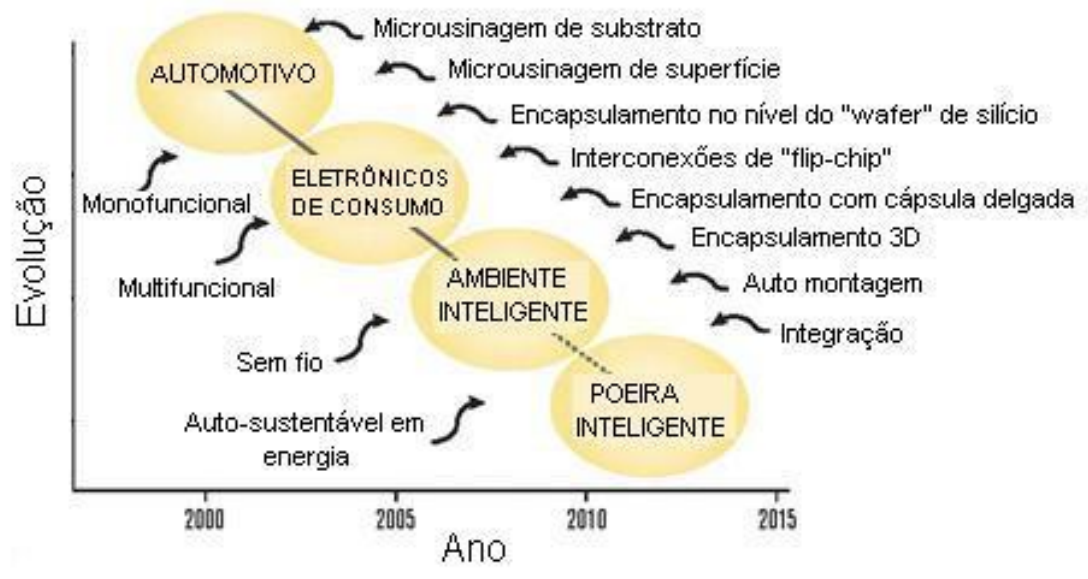

Figura 8: Avanços nos processos de fabricação de sensores MEMS e o surgimento de novas aplicações [22].

Os primeiros testes com dispositivos MEMS em sistemas aeroespaciais começaram na década de 1990 nas missões STS93 [28] e DS-2 [7]. Vários testes iniciais também foram conduzidos em meio acadêmico no desenvolvimento de satélites amadores [29]. Na primeira década do século 21, foi observada uma inserção rápida da tecnologia MEMS em sistemas aeroespaciais. Dispositivos MEMS já são usados como sensores e atuadores de altitude, no controle térmico e em antenas conforme ilustra a Figura 9. Contudo, há alguns desafios a serem superados no desenvolvimento de dispositivos para sistemas de propulsão, geração e armazenamento de energia [30].

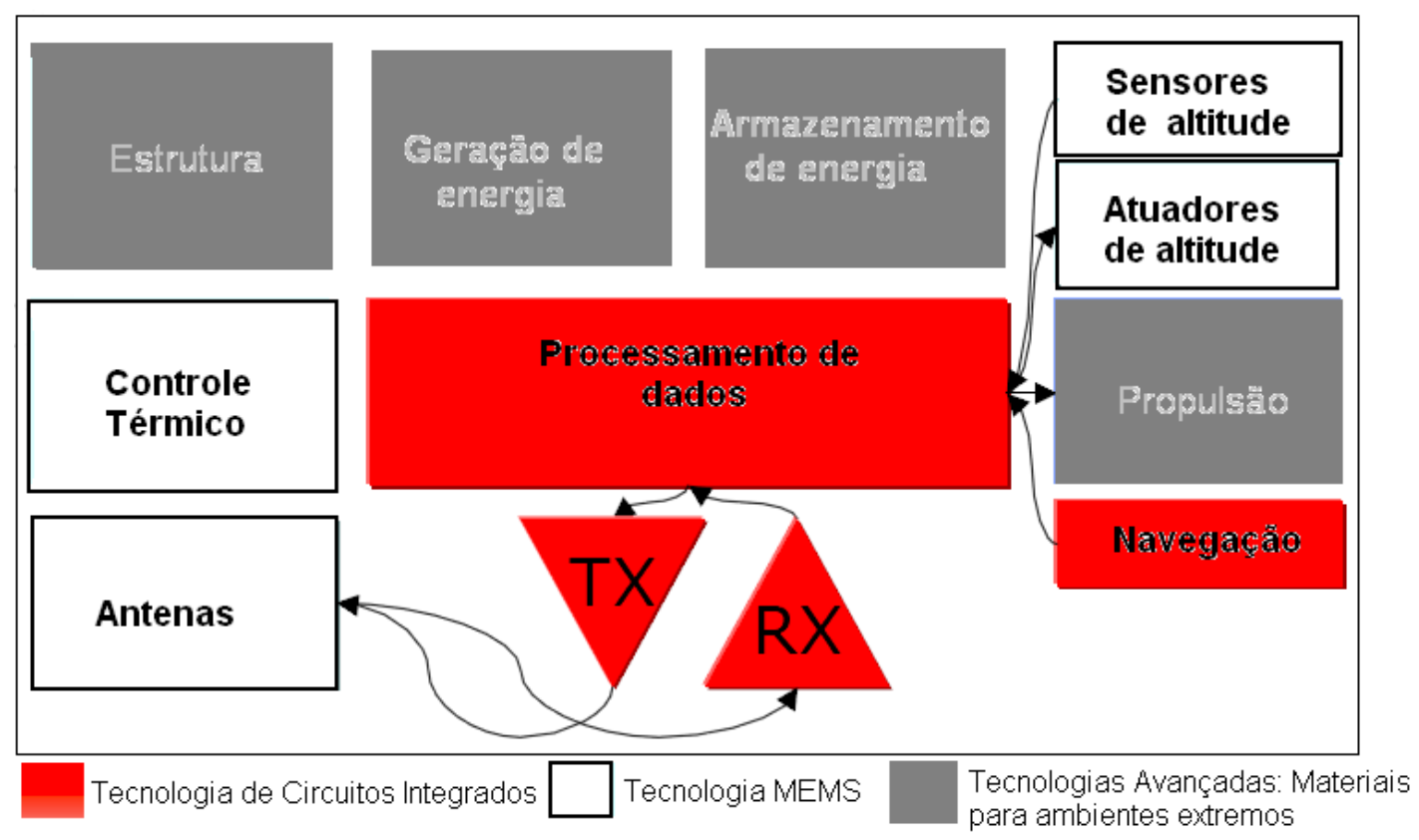

Figura 9: Tecnologias envolvidas nos elementos de um veículo espacial [무].

\section{EVOLUÇÃO DOS SENSORES MEMS PIEZORESISTIVOS BASEADOS EM SIC}

Os primeiros sensores baseados em $\mathrm{SiC}$ foram do tipo piezoresistivo, tal como os sensores de silício[19]. Isto porque elementos piezoresistivos são utilizados em muitas aplicações para fornecer sinal analógico para uma variedade de fenômenos físicos. Além disso, os sensores piezoresistivos apresentam uma configuração simples. A maioria desses sensores utiliza extensômetros ("strain gauges") de silício, configurados como ponte de Wheatstone, para medir parâmetros como 
pressão, aceleração e temperatura [19].

Em 1958, sensores de silício tipo "strain gauges" foram introduzidos no mercado para aplicações biomédicas. Este sensor apresentava uma estrutura simples: um "strain gauge" de silício colado sobre um diafragma metálico. Apenas no fim da década de 1960, surgiram tecno logias como corrosão anisotrópica e implantação iônica que possibilitaram a fabricação de extensômetros de silício sobre diafragma de silício [31].

Em 1967, a fabricante de sensores Endevco apresentou no $22^{\circ}$ Congresso Anual da Isa (" $22^{\text {nd }}$ Annual ISA Conference and Exhibit”), em Chicago, um relatório técnico descrevendo os sensores de pressão piezoresistivos que foram adaptados para o Programa Apollo coordenado pela NASA [32].

Entre as décadas de 1970 e 1980, a tecnologia de microfabricação foi aprimorada possibilitando o desenvolvimento de sensores cada vez menores, o que culminou com o surgimento dos primeiros sensores MEMS de silício [33]. Desde então, a tecnologia MEMS vem se consolidando e no estágio atual sabe-se que o seu desenvolvimento está diretamente associado com os avanços das técnicas de obtenção e de processamento de materiais para aplicação em ambientes extremos. Isto significa que a evolução da tecnologia MEMS depende principalmente de viabilizar a aplicação dos sensores onde o uso do silício é limitado, ou seja, produzir sensores com tamanho reduzido, baixo custo e capazes de suportar condições extremas de operação.

(a)

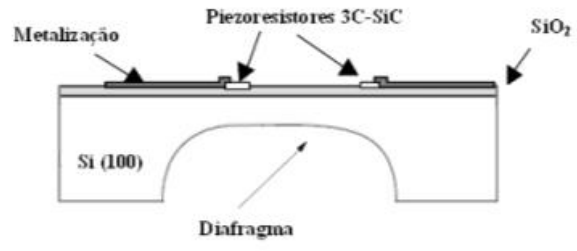

(b)

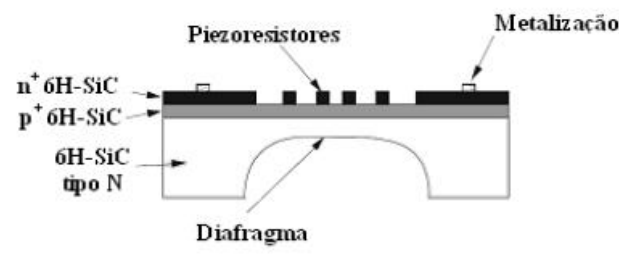

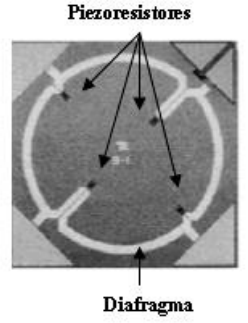

Contatos

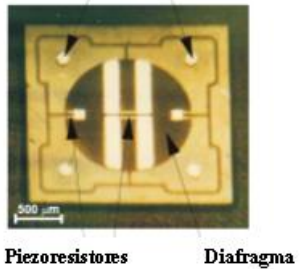

Figura 10: Sensores de pressão piezoresistivos baseados em SiC: (a) Universidade Técnica de Berlim (diafragma do sensor com $100 \mu \mathrm{m}$ de espessura) e (b) empresa Kulite Semiconductors (diafragma do sensor com $60 \mu \mathrm{m}$ de espessura)

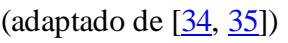

Os primeiros sensores MEMS piezoresistivos baseados em SiC foram desenvolvidos pela empresa Daimler-Benz em conjunto com a Universidade Técnica de Berlim [34]. Esse sensor foi construído a partir de um filme fino de 3C-SiC crescido sobre um substrato SOI (Silicon-On-Insulator). A estrutura do sensor é formada de quatro piezoresistores de 3C$\mathrm{SiC}$ sobre um diafragma de silício como ilustra a Figura 10 (a). Os testes realizados mostraram que o sensor apresentou um funcionamento estável em temperaturas de até $400^{\circ} \mathrm{C}$. Contudo, problemas decorrentes da baixa uniformidade do filme de 3C-SiC obtido pela técnica de deposição por vapor químico (Chemical Vapor Deposition - CVD) e da alta densidade de defeitos na interface $\mathrm{SiC} / \mathrm{SiO}_{2}$ foram observados [34].

Em 2004, a empresa Kulite Semiconductor Products desenvolveu sensores de pressão piezoresistivos em substrato de $6 \mathrm{H}-\mathrm{SiC}$ com funcionamento estável em temperaturas de até $500^{\circ} \mathrm{C}$ [35]. O sensor é constituído por quatro piezoresistores de $6 \mathrm{H}-\mathrm{SiC}$ tipo-n sobre um diafragma circular de $6 \mathrm{H}-\mathrm{SiC}$ tipo-p conforme ilustrado na Figura 10 (b). Apesar de ser todo confeccionado em $\mathrm{SiC}$, o que o torna atrativo para aplicações em ambientes extremos, esses sensores são caros porque foram fabricados em lâminas ("wafers") com diâmetro de 2 polegadas, enquanto a maioria dos fabricantes de dispositivos de silício já utilizavam lâminas superiores a 6 polegadas. Além disso, o processo de fabricação é complexo, principalmente a etapa de corrosão do $6 \mathrm{H}-\mathrm{SiC}$ para a formação do diafragma.

Em 2009, o potencial de alguns tipos de sensores MEMS a base de SiC para uso em uma aeronave foi discutido por Senesky e colaboradores [10]. Neste artigo, o potencial da tecnologia de SiC é analisado para os sensores apresentados na Figura 11. 


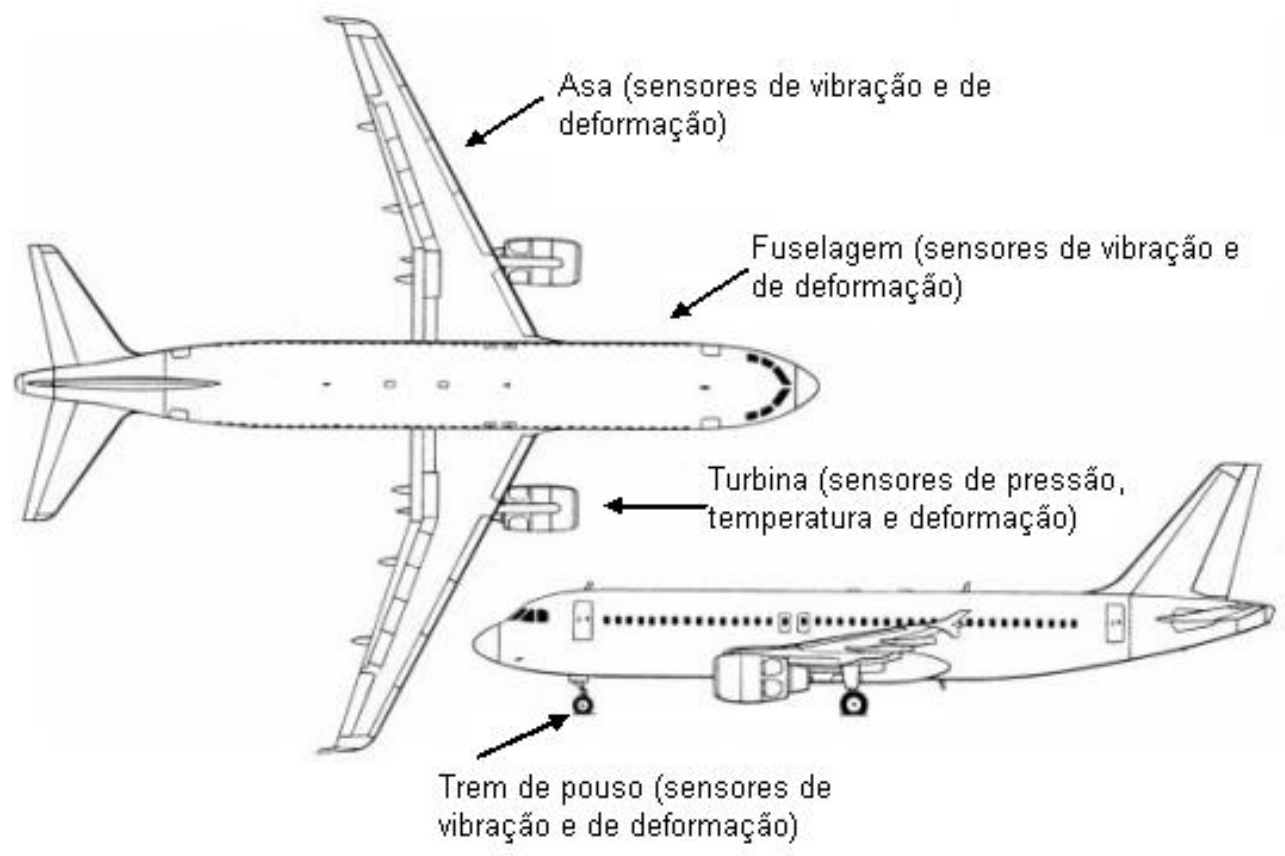

Figura 11: Aplicações de sensores MEMS em diferentes partes de uma aeronave (adaptado de [10]).

Atualmente, grupos de pesquisa acadêmicos e industriais de diversos países, destacando-se Estados Unidos e Alemanha, desenvolvem sensores baseados em substratos e filmes finos de SiC. Em geral, os sensores desenvolvidos têm mostrado bom desempenho em temperaturas de até $600^{\circ} \mathrm{C}$. No entanto, os substratos de $\mathrm{SiC}$ disponíveis comercialmente têm no máximo 3 polegadas de diâmetro e apresentam um custo em média quinze vezes maior que um de silício com as mesmas dimensões [36]. Além da redução de custos, os processos de corrosão são outro desafio dado que a estabilidade química do $\mathrm{SiC}$ o torna resistente a soluções químicas e a alguns gases comumente usados na corrosão do silício.

Os sensores baseados em filmes de $\mathrm{SiC}$ apresentam custo menor que aqueles desenvolvidos sobre substratos. Contudo, ainda exibem problemas de reprodutibilidade associados à baixa uniformidade do filme. Outro ponto relevante é que diversos estudos têm demonstrado que as características elétricas e mecânicas dos filmes finos de SiC variam significativamente em função do processo de deposição escolhido. Cabe ressaltar também que as técnicas de deposição dos filmes cristalinos utilizam altas temperaturas que são incompatíveis com os processos de microfabricação. O desafio é obter em baixa temperatura um filme de $\mathrm{SiC}$ com propried ades adequadas para construção de sensores MEMS.

No Brasil, as pesquisas sobre sensores piezoresistivos de SiC iniciaram-se em 2005 e em 2009 foi desenvolvido o primeiro protótipo de um sensor de pressão baseado em filme fino amorfo de SiC usando a técnica de PECVD (Plasma Enhanced Chemical Vapor Deposition) [19].

\section{PESQUISA E DESENVOLVIMENTO NO BRASIL E NO MUNDO DE SENSORES MEMS BASEADOS EM FILMES FINOS DE SIC}

Segundo dados do ISI Web of Knowledge, o desenvolvimento científico em dispositivos MEMS baseados em substratos ou filmes finos de $\mathrm{SiC}$ apresentou um rápido crescimento desde a sua apresentação à comunidade científica na década de 1990, conforme pode ser observado no gráfico apresentado na Figura 12. O número total de artigos para consulta com as palavras-chave "SiC + MEMS" foi de 462, enquanto que para as palavras-chave "SiC + MEMS + thin film" foi de 159. 


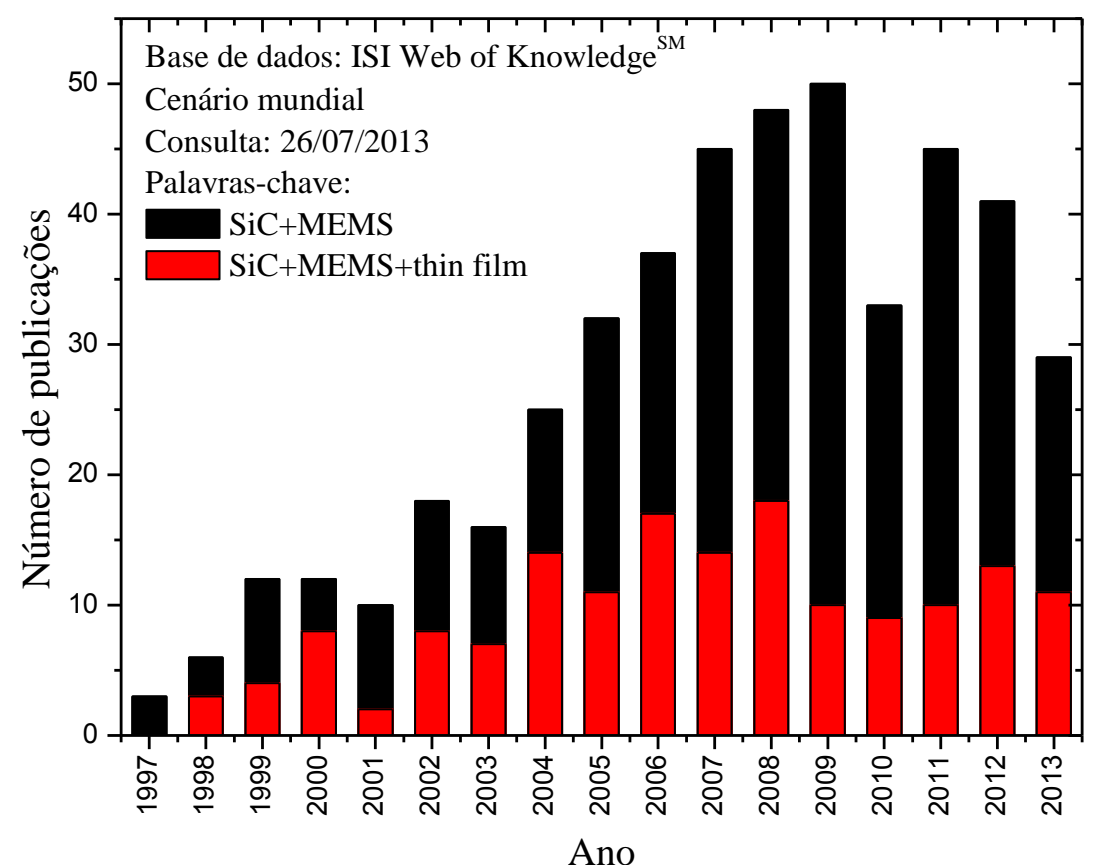

Figura 12: Número de publicações científicas relacionadas a dispositivos MEMS baseados em SiC no cenário mundial.

Entretanto, o cenário brasileiro não apresenta um desempenho similar. Em comparação com diversos países, sejam esses emergentes ou desenvolvidos, o Brasil está numa posição desfavorável (apenas 2,8\% do total de 159 artigos publicados no período de 1997-2013) apesar de sua economia emergente com recursos disponíveis para investimento neste tipo de tecnologia (veja Figura 13).

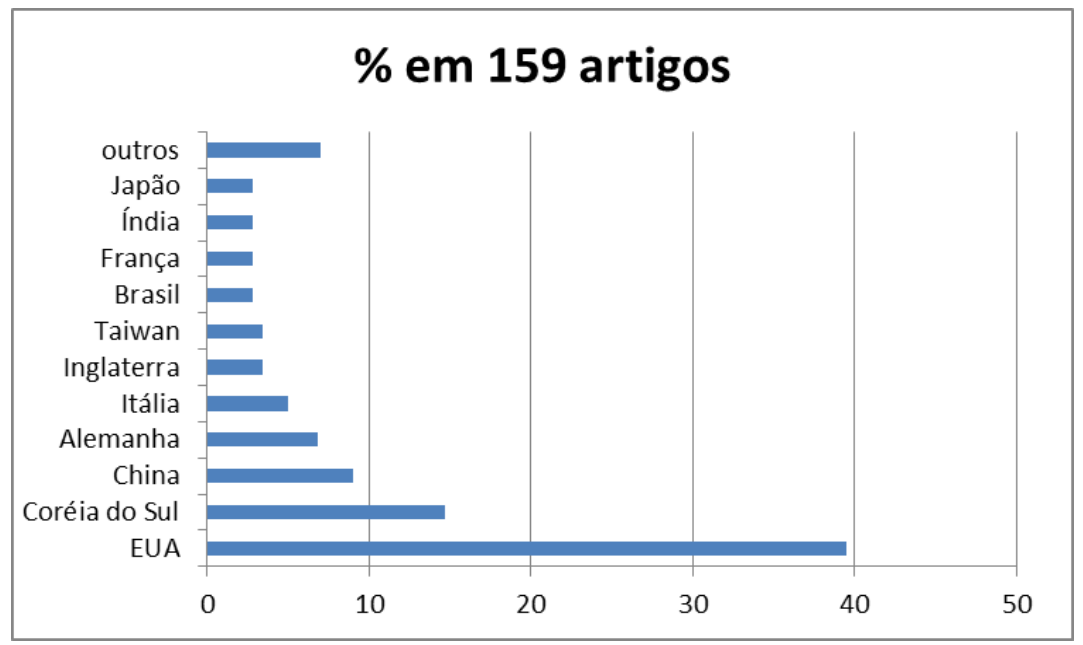

Figura 13: Ranking dos países que mais contribuem para o desenvolvimento de dispositivos MEMS baseados em filmes finos (thin films) de SiC. Palavras-chave: "SiC + MEMS + thin film".

Oficialmente, o Brasil passou a investigar cientificamente dispositivos MEMS baseados em SiC a partir dos anos 2000 (veja Figura 14). Embora tenha se passado mais de uma década desde o início dos trabalhos em solo nacional, pouco tem sido realizado cientificamente e industrialmente seja pelo fato de existirem poucos grupos de pesquisa efetiva no Brasil ou por outros fatores como, por exemplo, a falta de incentivo fiscal e/ou investimentos em Pesquisa e Desenvolvimento (P\&D) ou simplesmente pela baixa divulgação da tecnologia, uma vez que o mercado de MEMS é dominado por importações. 


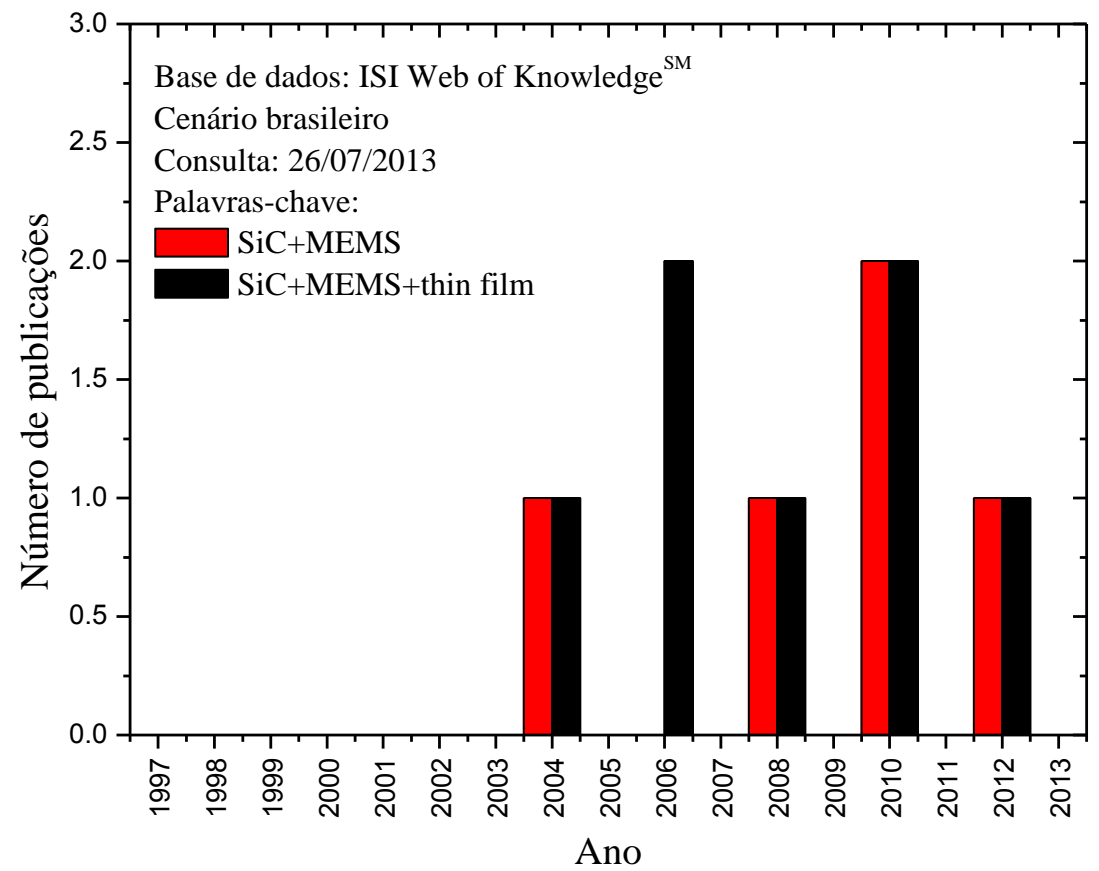

Figura 14: Número de publicações científicas relacionadas a MEMS baseados em SiC e ao material SiC na forma de filme fino no cenário brasileiro.

Dentre as principais instituições brasileiras que investem cientificamente em P\&D de dispositivos MEMS estão a Universidade de São Paulo (USP), o Instituto Tecnológico de Aeronáutica (ITA), o Instituto de Estudos Avançados (IEAv), a Universidade de Campinas (Unicamp) e o Laboratório Nacional de Luz Sincrotron (LNLS). Basicamente, P\&D em MEMS está centralizada na região sudeste do Brasil [37-41]. Nas buscas realizadas em bancos de dados de artigos em revistas indexadas não foi encontrada menção a apoio advindo de empresas estatais/privadas para este tipo de linha de pesquisa.

As principais agências brasileiras financiadoras de projetos envolvendo P\&D em MEMS são o Centro Nacional de Desenvolvimento Científico e Tecnológico $(\mathrm{CNPq})$, a Fundação de Amparo à Pesquisa do Estado de São Paulo (FAPESP) e a Coordenação de Aperfeiçoamento de Pessoal do Nível Superior (CAPES).

Em geral, os sensores MEMS desenvolvidos nos laboratórios brasileiros servem como "prova-de-conceito", ou seja, confirmam o potencial de um material ou técnica para aplicação neste tipo de dispositivo. Esses estudos não resultam no desenvolvimento de protótipos comercializáveis tanto pela falta de reprodutibilidade quanto pelo alto custo de produção e manutenção dos equipamentos necessários. Diante disso, as Universidades e Institutos de pesquisa nacionais têm focado seus esforços, principalmente, na síntese e caracterização de materiais para aplicações em sensores MEMS e na simulação destes sensores.

Estabelecer uma metodologia de produção de protótipos nacionais de sensores MEMS baseados em SiC e testá-los em aplicações aeroespaciais ainda é um desafio a ser superado. A Figura 15 ilustra todas as etapas envolvidas na produção do protótipo desde a síntese do filme fino de SiC até o encapsulamento. No Brasil, a realização de todas etapas só é possível utilizando a infraestrutura de laboratórios de diferentes instituições, o que além de dificultar a execução das etapas compromete bastante a qualidade dos dispositivos por não permanecerem em sala limpa durante todo processo de produção. 


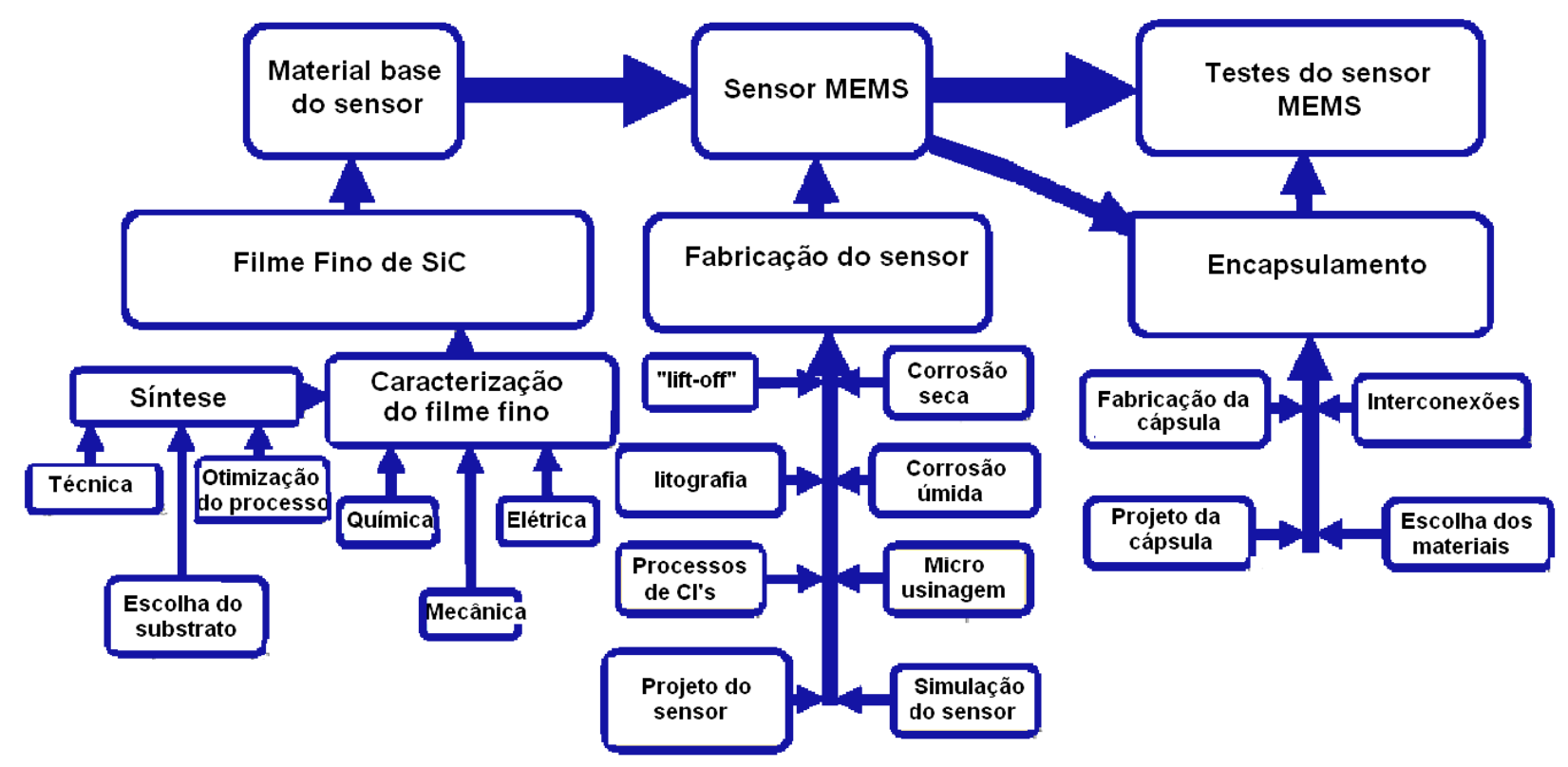

Figura 15: Etapas envolvidas na produção de sensores MEMS baseados em filmes de SiC.

\section{PERSPECTIVAS}

A indústria aeroespacial tem se beneficiado de novas tecnologias que permitem o projeto de veículos mais leves, com menor consumo de combustível, redução de emissões e diminuição dos ciclos de manutenção. Apesar dos recentes avanços, ainda são necessários desenvolvimentos na área de sensoriamento, em duas principais frentes: (i) sensores embutidos em materiais compósitos para a detecção de tensões localizadas e de trincas estruturais e (ii) sensores para monitoramento de ambientes extremos em tempo real [미].

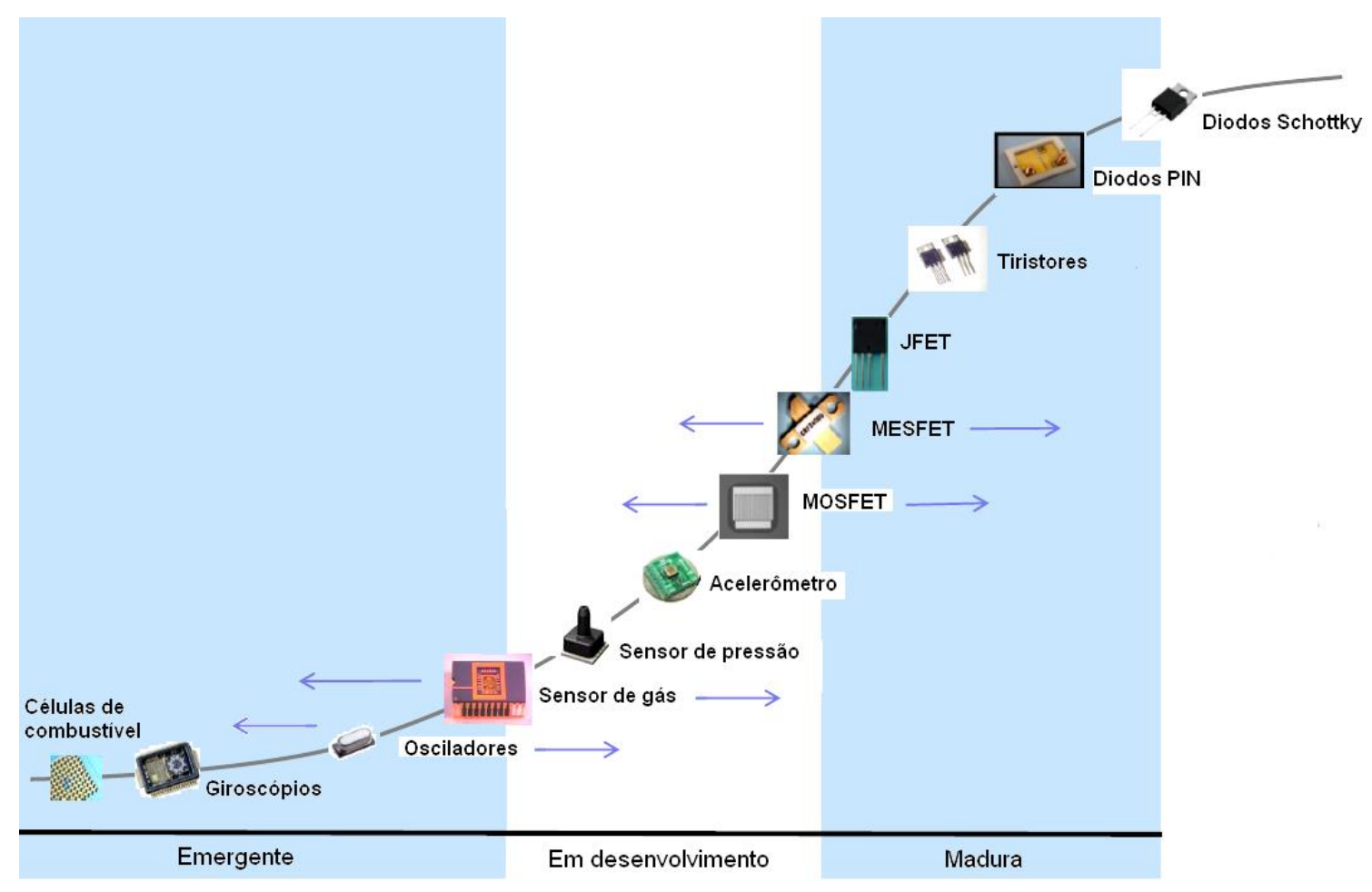

Figura 16: Estágio atual de desenvolvimento de dispositivos eletrônicos e sensores baseados em $\mathrm{SiC}$ (adaptado de [19]). 
A tecnologia de sensores MEMS baseados em SiC tem se mostrado atraente para uso em aplicações aeroespaciais e é forte candidata a se consolidar como uma tecnologia para ambientes extremos. Embora tenha havido recentes progressos na obtenção de substratos, com baixa densidade de defeitos além de menor custo, e de filmes finos com propriedades otimizadas para aplicação em sensores, a tecnologia de $\mathrm{SiC}$ ainda não está amadurecida o suficiente principalmente na parte de integração do sensor em sistemas aeroespaciais e da sua operação a longo prazo. A Figura 16 ilustra o estágio atual de desenvolvimento de dispositivos eletrônicos e sensores MEMS baseados em SiC. Observa-se que a tecnologia de dispositivos eletrônicos, diodos e transistores, baseados em SiC já está amadurecida, enquanto que a de sensores de pressão e acelerômetro encontra-se em desenvolvimento e os dispositivos emergentes são os giroscópios e as células de combustível.

As pesquisas realizadas no Brasil sobre filmes amorfos de $\mathrm{SiC}$ obtidos por processos assistidos a plasma, embora ainda estejam em estágio de amadurecimento, têm contribuído com avanços tanto na síntese do filme com propriedades otimizadas quanto na implementação dos sensores, com apoio das principais agências governamentais de apoio à pesquisa, além da Agência Espacial Brasileira, através do Programa Uniespaço. Os protótipos desenvolvidos, apesar de apresentarem problemas de reprodutibilidade, têm desempenho similar aos baseados em substratos e em filmes cristalinos de SiC. Muitos esforços têm sido feitos para solucionar esses problemas para que, num futuro próximo, os sensores de pressão e acelerômetros desenvolvidos possam ser testados em aplicações como em turbinas estacionárias a gás.

\section{AGRADECIMENTOS}

Os autores agradecem o auxílio financeiro da Agência Espacial Brasileira (AEB) através do Programa Uniespaço, da FAPESP (PRONEX 2011/50773-0, da CAPES e do CNPq.

\section{BIBLIOGRAFIA}

[1] GERSHMAN, R., WALLACE, R.A., "Technology Needs of Future Planetary Missions", Acta Astronautica, v. 45, pp. 329-335, 1999.

[2] SHERRIT, S., "Smart material/actuator needs in extreme environments in space". Proceedings of the SPIE Smart Structures Conference, San Diego, 2005.

[3] OSIANDER, R, ARRIN, M.A., CHAMPION, J.L., "MEMS and microstructures in aerospace applications", CRC Press, 2006.

[4] MOUNIER, E., "MEMS Markets \& Applications Focus on Wireless Sensor Networks \& Energy Harvesting", $2^{\text {nd }}$ Workshop on design, control and software implementation for distributed MEMS, Besancon, France, 2012.

[5] NEUDECK, P.G., OKOJIE, R.S., CHEN, L., "High-Temperature Electronics-A Role for Wide Bandgap Semiconductors?”, Proceedings of the IEEE, v. 90, 2002.

[6] PISANO, A.P., "Harsh Environment Sensor Cluster for Infrastructure Monitoring Single-Chip”, SelfPowered, Wireless, palestra ministrada na University of California, 2008.

[7] REYNOLDS, J.K., CATLING, D., BLUE, R.C., et al., "Packaging a piezoresistive pressure sensor to measure low absolute pressures over a wide sub-zero temperature range", Sensors and Actuators, v.83, pp.142-149, 2000.

[8] NESE, M., SEEBERG, B.E., LANG, M., "Silicon MEMS Pressure Sensor for Space", European Space Components Information Exchange System, 2005.

[9] NAJAFI, K. "Micropackaging Technologies for Integrated Microsystems: Applications to MEMS and MOEMS”, Proceedings of SPIE Micromachining and Microfabrication Process Technology, 2003.

[10] SENESKY, D.G., JAMSHIDI, B., CHENG, K.B., et al., "Harsh Environment Silicon Carbide Sensors for Health and Performance Monitoring of Aerospace Systems: A Review", IEEE Sensors Journal, v. 9, pp. 1472-1478, 2009.

[11] MATTINGLY, M., "Sensors for extreme environments require special design considerations", Test Engineering \& Management, pp. 12-14, 2010.

[12] CASADY, J.B., JOHNSON, R.W., "Status of Silicon Carbide (SiC) as a Wide-bandgap Semiconductor for High-Temperature Applications: A Review”, Solid-State Electronics, v. 39, pp. 1409-1422, 1996.

[13] SHEA, H.R., "Effects of Radiation on MEMS", Proc. of SPIE Reliability, Packaging, Testing, and Characterization of MEMS/MOEMS and Nanodevices, 2011. 
[14] EDMONDS, L.D., SWIFT, G.M., LEE, C.I., "Radiation Response of a MEMS Accelerometer: An Electrostatic Force", IEEE Transactions on Nuclear Science, v. 45, pp. 2779-2788, 1998.

[15] KNUDSON, A., BUCHNER, S., McDONALD, P., et al., "The Effects of Radiation on MEMS Accelerometers", IEEE Transaction on Nuclear Science, v. 43, pp. 3122-3126, 1996.

[16] SCHANWALD, L.P., WALSH, D.S., SMITH, N.F., et al., "Radiation effects on surface micromachined comb drives and microengines", IEEE Transactions on Nuclear Science, v. 45, 2002.

[17] YEW, C.K., "Silicon Carbide ( $\mathrm{SiC}$ )-Based Sensors for Harsh Environment Applications", Jurutera, The Institution of Engineers, Malaysia p. 22-29, 2007.

[18] FOTI, G. "Silicon carbide: from amorphous to crystalline material", Applied Surface Science, v. 184, p. 20-26, 2001.

[19] FRAGA, M. A. "Desenvolvimento de sensores piezoresistivos de SiC visando aplicação em sistemas aeroespaciais", Tese de Doutorado, Instituto Tecnológico de Aeronáutica, São José dos Campos, Brazil (2009).

[20] RAJAB, S. M., OLIVEIRA, I. C., MASSI, M., et al., "Effect of the thermal annealing on the electrical and physical properties of $\mathrm{SiC}$ thin films produced by RF magnetron sputtering", Thin Solid Films, v. 515, p. 170-175, 2006.

[21] TAYLOR J.K., "Global Markets for Electronic Pressure Transmitters, Transducers, and ComponentLevel Sensors", Process Pressure Transmitters, 2006.

[22] SALOMON, P., HEEREN, V. H., "Structural Health Monitoring - An Opportunity for MicroNano?", $R$ \& $D$ magazine, v. 50, p.47, 2008.

[23] LUO, M., SONG, C., HERRAULT, F., et al., "Microfabricated Wireless RF Pressure Sensor Made Completely of Biodegradable Materials", Solid-State Sensors, Actuators and Microsystems Workshop, 2012.

[24] HSU, T., "Miniaturization - A paradigm shift in advanced manufacturing and education", International conference on Advanced Manufacturing Technologies and Education in the 21 st Century, 2002.

[25] PISTER, K.S.J., "Introduction to MEMS Design and Fabrication”, Berkeley Sensor and Actuator Center, 2000.

[26] MACDONALD, N. C., "Scream MicroElectroMechanical Systems", Microelectronic Engineering, v. 32, p. 49-73, 1996.

[27] BOUCHAUD, J., "MEMS Enters New Cycle of Double-Digit Growth", Relatório HS ISuppli Market Research, 2011.

[28] OSIANDER, R., DARRIN, M. A., CHAMPION, J. L., "MEMS and microstructures in aerospace applications", CRC Press, 2006.

[29] CAMPBELL, M.E., BOHRINGER, K.F., VAGNERS, J., "Intelligent Satellite Teams", Proposal to the NASA Institute for Advanced Concepts, 1998.

[30] CURIEL, A.S., DAVIES, P., B AKER, A., et al., “Towards Spacecraft-on-a-Chip”, Surrey Space Centre, 2010.

[31] SANCHEZ, J.C., "Semiconductor strain-gauge pressure sensors", Instruments and Control Systems, p. 117-120, 1963.

[32] WALL, W.E., "Applications of Piezoresistance to Externally Excited Transducers", $22^{\text {nd }}$ Annual ISA Conference and Exhibit, Chicago, 1967.

[33] SUGYAMA, S., "Micro-diaphragm pressure sensor", International Electron Devices Meeting (IEDM), p.184-187, 1986.

[34] KROTZ, G., "New harsh environment sensor designs based on silicon carbide", MST News, v. 21, p. 17, Sep. 1997.

[35] NED, A.A., KURTZ, A.D., BEHEIM, G., et al., "Improved SiC Leadless Pressure Sensors For High Temperature, Low and High Pressure Applications", catálogo Kulite Semiconductors Products, 2004.

[36] LOBODA, M., "Design Considerations for SiC-based Power Electronics", Power and Electronics Technology for Designers and Systems Engineers, v. 38, p. 6, 2012.

[37] FRAGA, M.A., PESSOA, R.S., MACIEL, H.S., et al., "Recent developments on silicon carbide thin films for piezoresistive sensors applications", In: Moumita Mukherjee. (Org.). Silicon Carbide. Rijeka: Intech - Open Acess Publisher, v. 1, p. 369-388, 2011. 
[38] FRAGA, M.A., PESSOA, R.S., MACIEL, H.S., et al., "Technology Roadmap for Development of SiC Sensors at Plasma Processes Laboratory”, Journal of Aerospace Technology and Management, v. 2, p. 219224, 2010.

[39] FRAGA, M.A., FURLAN, H., PESSOA, R.S., et al., "Wide Bandgap Semiconductor Thin Films for Piezoelectric and Piezoresistive MEMS Sensors Applied at High Temperatures: An Overview", Microsystem Technologies, v. 20, p. 9-21, 2014.

[40] FRAGA, M. A., FURLAN, H., PESSOA, R.S., et al., "Studies on SiC, DLC and $\mathrm{TiO}_{2}$ thin films as piezoresistive sensor materials for high temperature application", Microsystem Technologies, v. 18, n. 7-8, p. 1027-1033, 2012.

[41] CARREÑO, M.N.P., LOPES, A.T., "Self-Sustained Bridges of a-SiC:H films obtained by PECVD at low temperatures for MEMS applications", Journal of Non-Crystalline Solids, v. 338, p. 490-495, 2004. 